\title{
Efficiency Assessment of Thermoelectric Generators in Heat-Insulated Combustion Engines
}

\author{
Alexey S. Osipkov, Mstislav O. Makeev, Yury P. Prilepo, and Georgy A. \\ Arutyunyan
}

Bauman Moscow State Technical University, Moscow, Russia

Keywords: Thermoelectric generator, combustion engine, efficiency, heat-insulated engines, effectiveness.

\begin{abstract}
In the present paper the efficiency of thermoelectric generators for different types of combustion engines was analyzed. Modeling of thermal flows through the thermoelectric generator and its output parameters at different placement from output collector of a standard and heatinsulated diesel engine was carried out. As the consequence, the perspective of the thermoelectric generators usage for heat-insulated combustion engines was shown. Thermoelectric generator for heat-insulated engine has similar physical specifications and several times as large power as compared with conventional engine. The possibility of reaching the efficiency up to $10 \%$ of the thermoelectric generators in heat-insulated engines by augmentation of heat transmission from exhaust to the junctures of thermoelectric battery was shown.
\end{abstract}

\section{Introduction}

Nowadays, combustion engines (CE) are the main source of energy for many vehicles, which generate about $90 \%$ of total power of the transport power systems. An annual increasing amount of vehicles imposes high economic and ecological requirements on piston engines that is why the problem of efficiency improvement of the $\mathrm{CE}$ becomes more significant.

In the modern engines a part of combusted fuel energy, which is being wasted on the engine net power, is approximately $35 \%$.

Other part of energy is wasted generally on the heat, which is carried away by coolant and exhaust. It has a lot of potential as in efficiency improvement of the engine workflow, as well as in recycling of the thermal losses.

There are many ways to improve the efficiency of the piston engines: using an alternative fuels, heat insulation of the combustion chamber, recycling of the exhaust power, recycling of the thermal losses. However, nowadays, the greatest interest are the exhaust power recycling, which can be implemented by means of the Rankine cycle and Stirling cycle engines, turbochargers or turbo compounding schemes, turbo- and thermoelectric generators.

One of the disadvantages of methods, based on the kinetic or thermal energy transformation of exhaust into mechanical energy, are the technical complexity of the mechanical energy usage and absence of the effective energy storage methods. The systems with an additional cycle use the transformation of mechanical energy into electrical energy, however, re-converting decreases the total effectiveness of the system, and the necessity of electric generator installation increases the cost and mass of the system.

The most promising method of increasing the energy effectiveness of vehicles on the CE is the direct transformation of a heat, released by exhaust, into electricity by thermoelectric generator batteries (TEB), which have unique properties, such as high reliability, high battery life, they are not depend on spatial placement and environment.

The limiting factors of using thermoelectric transformation method of thermal energy into electrical energy in the $\mathrm{CE}$ are low effectiveness of the nowadays used thermoelectric materials and high its cost. It can be shown, that optimization of the thermoelectric generator (TEG) construction in conjunction with the other ways of effectiveness improvement of piston engines, which lead to 
expansion of the temperature gradient located at the junctures of the TEB, may significantly improve as the efficiency of the TEG, as well as the effectiveness of the engine in general.

In this work, the effectiveness of the thermoelectric generators for different types of combustion engines was analyzed. Modeling of thermal flows through the thermoelectric generator and its output parameters at different placement from the output collector of a standard and heat-insulated diesel engine was carried out.

\section{Results and Discussion}

While modeling of thermal flows and output parameters of the TEG, it can be represented as the multilayered pipe, which corresponds to an installation of the generator with the ring thermoelectric batteries (TEB). At the flat TEB usage, the TEG is often formed as the polyhedron; however, it can be replaced with a high precision by a tube of a round cross-section with an equivalent diameter.

Modeling scheme is shown in Fig. 1. The initial data for modeling are power of the engine, temperature and thermal flow of exhaust, outer dimensions of the TEG, physical properties of the thermoelectric semiconductor materials used in the construction of the TEB, and the thermodynamic properties of materials used in the construction.

To assess the effectiveness of the TEG there were solved the problems of a heat transfer through the cylindrical wall and heat exchange between exhaust and a flat wall.

The output parameters modeling of the TEG was performed as described in [1] for two types of engines: a powerful diesel engine TMZ 8463.10 of a lorry and a gasoline engine of a passenger car Lada Kalina (21127). While modeling, a diesel engine consisted of a low temperature material based on $\mathrm{Bi}_{2} \mathrm{Te}_{3}$, and a gasoline engine consisted of a medium temperature materials based on PbTe and GeTe (see Table 1) [2].

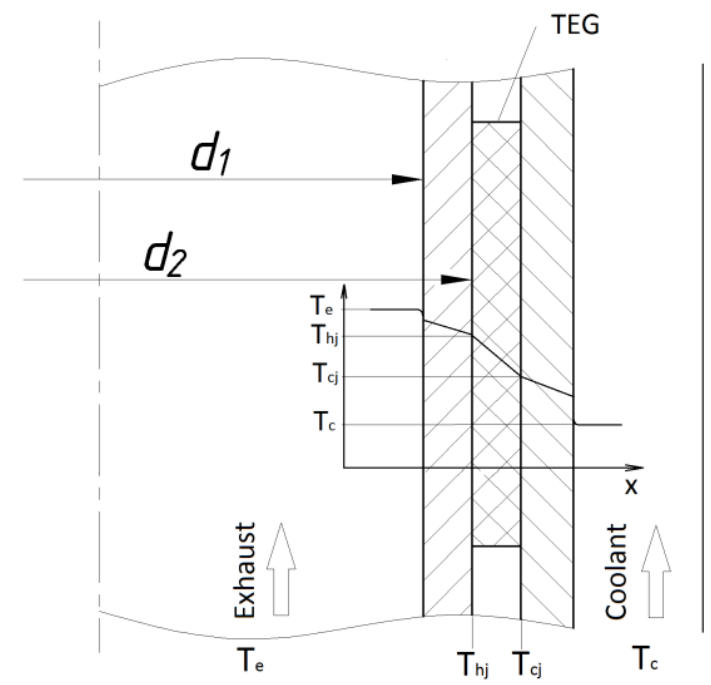

Fig. 1: Modeling scheme of the TEG

Table 1: Properties of the Thermoelectric Materials

\begin{tabular}{llllll}
\hline $\begin{array}{l}\text { Mate- } \\
\text { rial }\end{array}$ & $\begin{array}{l}\text { Type } \\
\text { of } \\
\text { condu- } \\
\text { ctivity }\end{array}$ & $\begin{array}{l}\text { Operating } \\
\text { tempera- } \\
\text { ture } \\
\text { range, }\end{array}{ }^{\circ} \mathbf{C}$ & $\begin{array}{l}\text { Seebeck } \\
\text { coefficient } \\
\boldsymbol{\alpha} \mathbf{V} / \mathbf{K}\end{array}$ & $\begin{array}{l}\text { Resistivity } \\
\boldsymbol{\rho},\end{array}$ & $\begin{array}{l}\text { Coefficient } \\
\text { Ohm } \cdot \mathbf{c m} \text { thermal } \\
\text { conductivity } \\
\mathbf{\kappa}, \\
\mathbf{W} /(\mathbf{c m} \cdot \mathbf{K})\end{array}$ \\
\hline $\mathrm{Bi}_{2} \mathrm{Te}_{3}$ & $n$ & $20-300$ & $171,0 \cdot 10^{-6}$ & $9,29 \cdot 10^{-4}$ & $2,0 \cdot 10^{-2}$ \\
\hline $\mathrm{Bi}_{2} \mathrm{Te}_{3}$ & $p$ & $20-300$ & $165,3 \cdot 10^{-6}$ & $12,0 \cdot 10^{-4}$ & $2,0 \cdot 10^{-2}$ \\
\hline $\mathrm{PbTe}$ & $n$ & $200-500$ & $187,1 \cdot 10^{-6}$ & $30,91 \cdot 10^{-4}$ & $1,4 \cdot 10^{-2}$ \\
\hline $\mathrm{GeTe}$ & $p$ & $200-500$ & $225,4 \cdot 10^{-6}$ & $21,37 \cdot 10^{-4}$ & $1,5 \cdot 10^{-2}$ \\
\hline
\end{tabular}


In Table 2 shown the results of modeling for viewed engines at the TEG, which had a length of $0,5 \mathrm{~m}$, placement near the output collector.

Despite of the fact that the thermal flow power of exhaust at the output collector of the engine TMZ 8463.10 has 3,7 times as large power as appropriate power of the engine Lada Kalina (21127), an output power of the TEG for a lorry was 1,5 times low due to lower thermal flow, which comes through the TEG, and lower efficiency of the thermoelectric conversion.

Let us consider the possible variants of effectiveness improvement of the TEG on a diesel engine $\eta$, which can be represented as [3]:

$$
\eta=\frac{T_{h}-T_{c}}{T_{h}} \frac{M-1}{M+\left(T_{c} / T_{h}\right)},
$$

where $T_{h}$ and $T_{c}$ - the temperatures of a hot and cold junctures of the TEG, respectively, K; $M-$ optimum for a maximum efficiency mode of a payload $R_{p}$ divided by internal resistance $r_{T E G}$, which characterizes a measure of irreversible losses in thermocouple.

$$
M=R_{p} / r_{T E G}=\sqrt{1+1 / 2 Z\left(T_{h}+T_{c}\right)},
$$

where $Z$ - thermoelectric figure of merit, a complex criterion, which determines the quality used thermoelectric material, $K^{-1}$.

Table 2: The Results of the TEG Parameters Modeling

\begin{tabular}{ccc}
\hline Parameter & $\begin{array}{c}\text { Gasoline engine of a } \\
\text { passenger car Lada } \\
\text { Kalina (21127) }\end{array}$ & $\begin{array}{c}\text { Diesel engine } \\
\text { TMZ 8463.10 } \\
\text { of a lorry }\end{array}$ \\
\hline Engine power, $\mathrm{kW}$ & 77,9 & 367,6 \\
\hline Thermal flow of exhaust, $\mathrm{kW}$ & 78,6 & 290,5 \\
\hline Temperature of exhaust, ${ }^{\circ} \mathrm{C}$ & 800 & 600 \\
\hline Thermal flow through the TEG, $\mathrm{kW}$ & 16,2 & 11,9 \\
\hline $\begin{array}{c}\text { Temperature difference at the } \\
\text { junctures of the TEG, } \mathrm{K}\end{array}$ & 200 & 148 \\
\hline Output power of the TEG, $\mathrm{W}$ & 626 & 404 \\
\hline Efficiency, $\%$ & 4,5 & 3,8 \\
\hline Mass of the TEB, $\mathrm{kg}$ & 5,20 & 4,57 \\
\hline
\end{tabular}

The first multiplier of (1) represents the efficiency of reversible process (the Carnot cycle). The second multiplier represents the efficiency decreasing as the result of irreversible losses of a thermal conductance and Joule heat.

Thermoelectric figure of merit can be expressed as:

$$
Z=\alpha^{2} \sigma / \kappa,
$$

where $\alpha$ - thermopower coefficient, $\mathrm{V} / \mathrm{K} ; \sigma$ - electrical conductivity, $\kappa$ - thermal conductivity, $\mathrm{W} /(\mathrm{cm} \cdot \mathrm{K})$.

Analyzing (1-3), the two main ways of the TEG effectiveness improvement can be distinguished:

- improvement of thermoelectric materials figure of merit;

- extension of temperature difference between TEB junctions.

The second way can be achieved by means of the CE mode changing, decreasing the thermal and electrical resistances of the TEB switching layers, decreasing the thermal resistance of the thermal contact junctures, and optimization of the thermocouple constructions used in the TEG by applying an advanced layout drawings of the thermocouple branches with its cascading or segmentation.

Moreover, the temperature difference expansion inevitably entails the temperature increasing of a hot juncture, which in turn lead to increasing of the thermal flow through the TEG.

One of the promising ways of the CE mode changing, which leads to the TEG effectiveness increasing, is its heat insulation [4]. The idea of the heat-insulated engine consist in creating a 
combustion chamber with a heat-resistant and heat-insulated walls, which have exactly the same transient temperature as the working medium at given time.

When insulating the combustion chamber of a heat load on the part of the working medium, it is possible to dissipate most of the heat, which is usually released by the water cooling system of the engine, into exhaust. However, the exhaust at the output of a heat-insulated engine will have more reserves of the enthalpy at the higher temperature that enables the effective usage of the energy recycling systems. Thus, the heat insulation usage enables to increase a part of a heat, which is expended on the useful work and released by exhaust, and decrease the thermal losses into the cooling liquid.

Modeling of the thermal balance for the standard and heat-insulated engine showed, that:

- a part of a heat released of the engine by the cooling medium for the heat-insulated engine is $17 \%$ ( $25 \%$ for the standard);

- a part of a heat released by exhaust for the heat-insulated engine increases from 29 to $35 \%$;

- a part of a heat which is equivalent to the effective engine performance for the heat-insulated engine is $48 \%$, which is $8 \%$ as large as the standard engine.

The results of modeling of the TEG parameters at its placement at different distances from the output collector of the standard and heat-insulated engine TMZ 8463.10 are shown in Fig. 2.

While modeling, the finning coefficient, which shows an increasing of the heat exchanger surface area in comparison with a smooth pipe, was equal to 3 . The calculations of the TEG parameters at the different variants of its placement in exhaust system of the engine showed that the power and efficiency of the TEB are decreased regularly with the distance of the generator from the output collector. The heat-insulated engine has $3 \ldots 4$ times as large power as conventional engine at the similar outer dimensions and weight.

The maximum of the TEG power can be achieved when the temperature difference at the TEG junctures is half of the maximum possible difference between the ambient temperature and the temperature of exhaust gases [3].

At the environmental temperature up to $50{ }^{\circ} \mathrm{C}$ and the temperature of exhaust at the output collector up to $600{ }^{\circ} \mathrm{C}$ the temperature difference for a diesel engine is $550{ }^{\circ} \mathrm{C}$. Then, the maximum efficiency and power of the TEG will be at the temperature difference on the junctures of the TEG at $275{ }^{\circ} \mathrm{C}$. In case of the heat-insulated engine at the exhaust temperature of $1100{ }^{\circ} \mathrm{C}$ at the output collector [4], the temperature difference at the junctures of the TEB will be $525^{\circ} \mathrm{C}$.

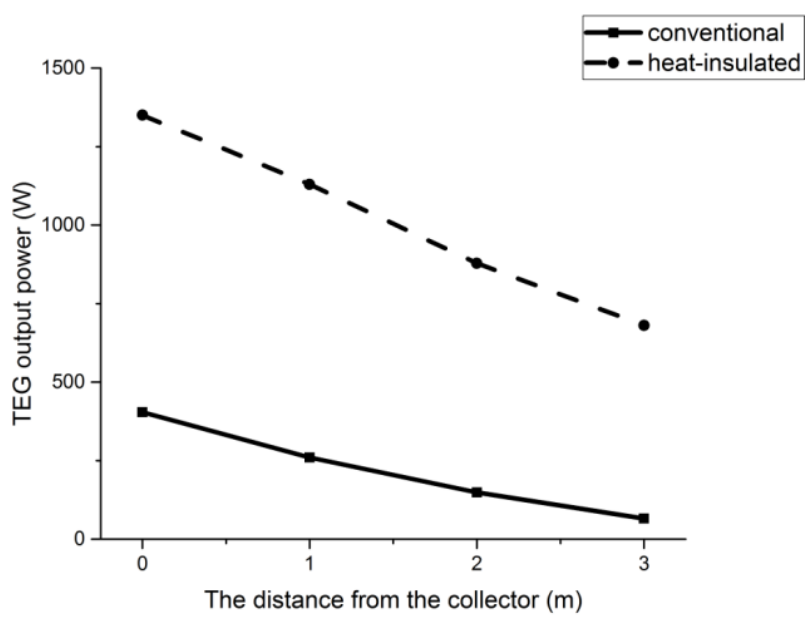

Fig. 2: Output power of the TEG versus TEG placement at the output collector of the standard and heat-insulated diesel engine.

To achieve required temperature difference, it is necessary to increase the temperature of a hot juncture by means of the intensification of a heat exchange between exhaust and the wall of the exhaust path.

In Fig. 3 shown the TEG power and its efficiency versus finning coefficient for the standard and heat-insulated engines TMZ 8463.10. 
To maximize the effectiveness of the TEG, the finning coefficient for viewed diesel engine has to be no less than 12. At the same time, the TEG power at its placement near the output collector for the standard and heat-insulated engines will be $1,38 \mathrm{~kW}$ and $5 \mathrm{~kW}$, respectively, and the efficiency will be $6,1 \%$ and $11 \%$, respectively.

Considering, that the finning coefficient increasing and, therefore, the heat exchange intensification between exhaust and the wall of the exhaust path increases the aerodynamic drag of exhaust which has a negative impact on the workflow of the $\mathrm{CE}$ and may level all the energy gain, which was achieved at the TEG installation. Accordingly, the problem of the heat exchange intensification between exhaust and the junctures of the TEB at the minimal aerodynamic drag to exhaust is very important.

Moreover, the temperature on a hot juncture of the TEB at the heat-insulation of the engine will be $800{ }^{\circ} \mathrm{C}$, which may require the usage of the segmented branches or cascaded thermocouples with the cascades (segments) of a low-, middle-, and high-temperature thermoelectric materials in the TEG.
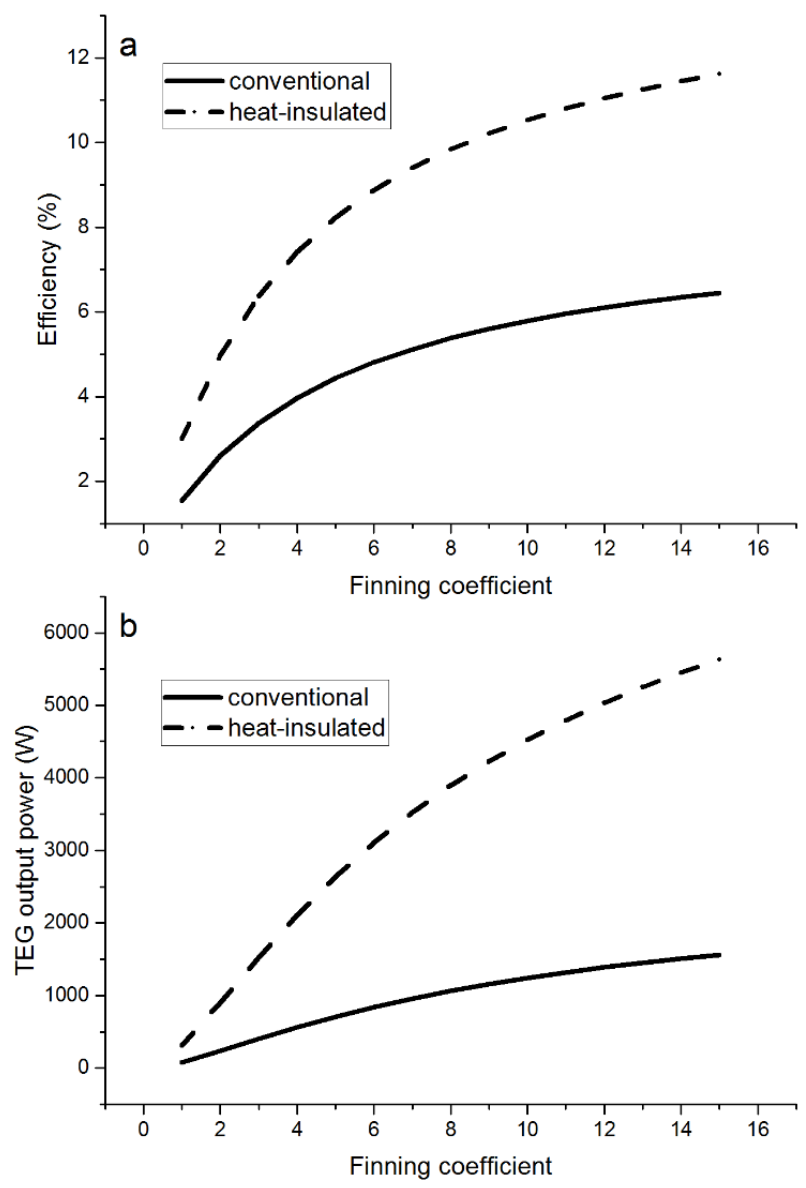

Fig. 3: Efficiency (a) and output power (b) of the TEG versus finning coefficient for the standard and heat-insulated diesel engines.

\section{Conclusions}

Modeling results of the thermal flows through the thermoelectric generator and its output parameters at the TEG placement at different distances from the output collector of the standard and heat-insulated diesel engine showed that the effectiveness of the TEG decreases with the distance from the output collector, therefore it is reasonable to place the TEG at the beginning of the exhaust system. The heat-insulated engine has 3...4 times as large power as conventional engine at the similar outer dimensions and weight. 
To maximize the effectiveness of the TEG, the finning coefficient of such diesel engine has to be no less than 12. At the same time, a power of the TEG on this diesel engine will be about $5 \mathrm{~kW}$ and efficiency of the battery will be above $10 \%$.

Installation of the TEG on the heat-insulated engine at the output collector before turbocharger will slightly decrease the temperature of exhaust. It may be a promising solution to the problem of using turbochargers, which have materials with a low thermal resistance, in these engines.

Modeled values of TEG power on the heat-insulated engine enable to consider the rejection of a regular electric generator, which will significantly increase a fuel effectiveness of the vehicle.

\section{Acknowledgements}

The research work was supported by Ministry of Education and Science of the Russian Federation (unique identifier RFMEFI57714X0113).

\section{References}

[1] O.V. Marchenko, A.P. Kashin, V.I. Lozbin, M.Z. Maksimov: Methods of calculation of thermoelectric generators (Nauka, Novosibirsk, 1995).

[2] L.D. Ivanova, L.I. Petrova, Yu.V. Granatkina, V.G. Leontyev, A.S. Ivanov, S.A. Varlamov, Yu.P. Prilepo, A.M. Sychev, A.G. Chuiko, I.V. Bashkov: Vol. 49, I. 2 (2013), p. 120.

[3] D.M. Rowe: Thermoelectrics Handbook: Macro to Nano (CRC Press, 2005).

[4] R. Kavtaradze, A. Zelentsov, S.P. Gladyshev, Z. Kavtaradze, D. Onishchenko: SAE Technical Paper 2012-01-1217 (2012), doi:10.4271/2012-01-1217. 